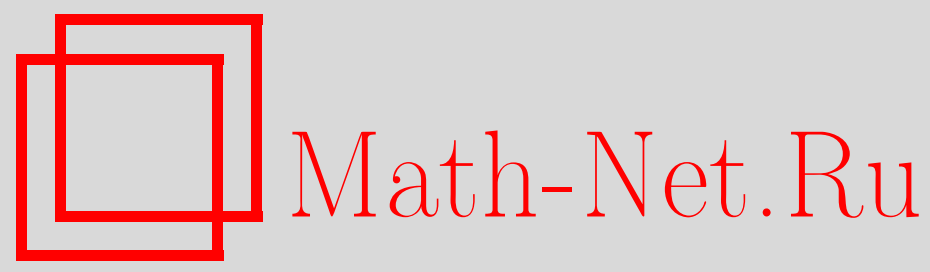

В. Ж. Сакбаев, О задаче Коши для уравнения среднего поля, описывающего модель твердого магнетика, Матем. заметки, 2001, том 70, выпуск 3, 434-445

DOI: https://doi.org/10.4213/mzm755

Использование Общероссийского математического портала Math-Net.Ru подразумевает, что вы прочитали и согласны с пользовательским соглашением http://www.mathnet.ru/rus/agreement

Параметры загрузки:

IP: 54.198 .64 .247

26 апреля 2023 г., $17: 41: 28$

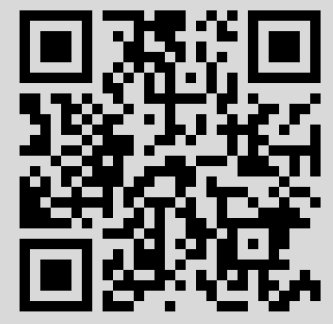




\title{
О ЗАДАЧЕ КОШИ ДЛЯ УРАВНЕНИЯ СРЕДНЕГО ПОЛЯ, ОПИСЫВАЮЩЕГО МОДЕЛЬ ТВЕРДОГО МАГНЕТИКА
}

\author{
В. Ж. Сакбаев
}

Рассматривается модель твердого магнетика как системы частиц, обладающих механическим моментом $\vec{s}, \vec{s} \in S^{2}$, и магнитным моментом $\vec{\mu}, \vec{\mu}=\vec{s}$, которые взаимодействуют друг с другом посредством магнитного поля, что определяет изменение механического момента каждой частицы. Изучается система интегродифференциальных уравнений, определяющая эволюцию одночастичной функции распределения указанной системы частиц. Доказаны теоремы о существовании и единственности обобщенного и классического решений задачи Коши для данной системы уравнений и о непрерывной зависимости обобщенного решения от начальньх условий.

Библиографояи: 6 названий.

1. Введение. Описание модели. В настоящей работе рассмотрена модель, описьвающая поведение магнетика, состоящего из частиц (атомов, молекул, ионов), обладающих ненулевьп спином и ненулевьм магнитным дипольньм моментом (МДМ). В рассматриваемой модели применяется классическое описание состояния и динамики системы: каждая частица вешества характеризуется своим положением в пространстве $\vec{x} \in \mathbb{R}^{3}$; ее спиновое состояние определяется 3 -мерньм вектором механического момента частицы $\vec{s}(t),|\vec{s}(t)|=1$, для каждой частицы (см. [1]-[3]).

Предполагается, что частица, находящаяся в состоянии $\vec{s}$, обладает МДМ $\vec{\mu}=\mu_{e} \vec{s}$, где константа $\mu_{e}$ определяется свойствами частиц. Пусть единицы измерения физических величин выбраны так, что $\mu_{e}=1$. МДМ $\vec{s}$, находящийся в точке $\vec{x}_{0} \in \mathbb{R}^{3}$, создает магнитное поле, равное

$$
\vec{B}(\vec{x})=\nabla_{x}\left(\frac{\left(\vec{s}, \vec{x}-\vec{x}_{0}\right)}{\left|\vec{x}-\vec{x}_{0}\right|^{3}}\right),
$$

в точках, достаточно удаленных от $\vec{x}_{0}$ (см., например, [3]).

Будем предполагать, что частицы вешества расположены в ограниченной области $\Omega \subset \mathbb{R}^{3}$ с гладкой границей $\partial \Omega$; тогда состояние каждой частицы характеризуется координатами $(\vec{x}, \vec{s}), \vec{x} \in \Omega, \vec{s} \in S^{2}$.

В данной модели предполагается, что каждая частища находится в некоторой точке $\Omega$ и не изменяет своего положения $\vec{x}$, а движение ее спиновой координаты $\vec{s}(\vec{x}, t)$ подчиняется классическому уравнению (см. [1], [2])

$$
\frac{d \vec{s}}{d t}=[\vec{B}(\vec{x}, \vec{s}, t) \times \vec{s}],
$$


где момент сил $[\vec{B} \times \vec{s}]$ возникает в результате взаимодействия МДМ с магнитным полем, созданным всей совокупностью МДМ и внешними источниками, а также в результате учета электростатического взаимодействия частиц друг с другом с помощью введения эффективных магнитньх полей. Здесь $\vec{B}(\vec{x}, \vec{s}, t)$ - индукция суммарного эффективного магнитного поля, которая в соответствии с [1], [2] определяется как суперпозищия внешнего магнитного поля $\vec{B}_{0}(\vec{x}, t)$, магнитного поля дипольного взаимодействия $\vec{B}(\vec{x}, t)$ и специальных полей, имеющих электростатическую природу: эффективного магнитного поля обменного взаимодействия $\vec{B}_{I}(\vec{x}, t)$ и эффективного магнитного поля анизатропии кристалла $\vec{B}_{a}(\vec{s})$. Возможность описать влияние электрических полей на изменение состояния частицы с помощью эффективного магнитного поля обсуждалась в указанной литературе. Вьводы работы справедливы, в частности, и для систем с чисто магнитным взаимодействием.

В статье изучается описание самосогласованной динамики спинового состояния системы $\vec{s}(\vec{x}, t)$ в поле $\vec{B}(\vec{x}, \vec{s}, t)$, связанном с этой динамикой. Рассматриваются вопросы корректности задачи Коши и связи предлагаемого описания непрерьвной среды с описанием многочастичной системы с помощью классических уравнений движения.

Состояние системы частищ в момент времени $t$ будем определять с помощью функции распределения в координатном пространстве $X=\Omega \times S^{2}: f(\vec{x}, \vec{s}, t)$ есть плотность вероятности распределения частиц в пространстве $X$ в момент времени $t$, т.е. если число частиц системы равно $N$, то для любой области $D \in X$ величина $N \int_{D} f(\vec{x}, \vec{s}, t) d x d s$ характеризует число частищ, находящихся в области $D$ пространства $X$ в момент времени $t$. По своему смыслу функция $f(\vec{x}, \vec{s}, t)$ должна удовлетворять следующим требованиям:

1) $f(\vec{x}, \vec{s}, t) \geqslant 0$ для всех $(\vec{x}, \vec{s}, t)$;

2) $\int_{X} f(\vec{x}, \vec{s}, t) d x d s=1$ при всех $t$.

Введем следующие обозначения.

1) $T_{\vec{s}}\left(S^{2}\right)$ - касательная плоскость к единичной сфере в точке $\vec{s}$. На сфрере $S^{2}$ выбран и фиксирован атлас из двух карт $\left\{\Sigma_{1}, \Sigma_{2}\right\}$, координаты в каждой из которых обозначаются через $\sigma_{1}, \sigma_{2}$; тогда $\vec{s}=\vec{s}\left(\sigma_{1}, \sigma_{2}\right)$. Выбор локальной системы координат $\left(\sigma_{1}, \sigma_{2}\right)$ в карте определяет в касательных плоскостях $T_{\vec{s}}\left(S^{2}\right)$ в каждой точке сферы базис $\vec{e}_{1}(\vec{s})$, $\vec{e}_{2}(\vec{s})\left(\vec{e}_{i}(\vec{s})=\left(\partial \vec{s}\left(\sigma_{1}, \sigma_{2}\right) / \partial \sigma_{i}\right)\left|\partial \vec{s} / \partial \sigma_{i}\right|^{-1}\right)$.

2) Пространство непрерьвно дифференцируемых $k$ раз в каждой точке сферы вещественнозначных функций обозначается через $C^{k}\left(S^{2}\right)$; введено обозначение

$$
D_{\sigma_{\alpha}}^{i} f(\vec{s})=\left(\frac{\partial}{\partial \sigma_{\alpha}}\right)^{i} f\left(\vec{s}\left(\sigma_{1}, \sigma_{2}\right)\right), \quad \alpha=1,2, \quad i=1, \ldots, k,
$$

если $f\left(\vec{s}\left(\sigma_{1}, \sigma_{2}\right)\right) \in C^{k}\left(S^{2}\right)$.

3) $C^{k}(X)$ - множество $k$ раз непрерьвно дифференцируемых функций на ограниченном замкнутом множестве $[X]$ (замыкание $X$ в $\mathbb{R}^{3} \times S^{2}$ ) с гладкой границей. Чтобы определить норму в пространстве $C^{k}(X)$, рассмотрим покрытие сферы меньшими картами $\left\{\Sigma_{1}^{\prime}, \Sigma_{2}^{\prime}\right\}$, лежащими строго внутри соответствующих карт исходного покрытия. Тогда определим норму в $C^{k}(X)$ равенством

$$
\begin{aligned}
& \left\|f(\vec{x}, \vec{s}) \mid C^{k}(X)\right\| \\
& \quad=\max _{a=1,2}\left(\sup _{l=0, \ldots, k}\left(\sup _{i_{1}+\cdots+j_{2}=l}\left(\sup _{\Omega \times \Sigma_{a}^{\prime}}\left|D_{x_{1}}^{i_{1}} D_{x_{2}}^{i_{2}} D_{x_{3}}^{i_{3}} D_{\sigma_{1}}^{j_{1}} D_{\sigma_{2}}^{j_{2}} f\left(\vec{x}, \vec{s}\left(\sigma_{1}, \sigma_{2}\right)\right)\right|\right)\right)\right),
\end{aligned}
$$


где $i_{1}, \ldots, j_{2}$ - неотрицательные целые числа. Нетрудно проверить, что данное равенство действительно определяет норму.

4) Пусть $Y$ - банахово пространство. Тогда $C^{i}([0, T] \times X, Y), i=0,1$, есть соответственно непрерывное и непрерывно дифференцируемое по совокупности аргументов $t, \vec{x}$, $\vec{s}$ отображение пространства $[0, T] \times X$ в пространство $Y$.

5) Для функций $f(\vec{s})$ из $C^{1}\left(S^{2}\right)$ и для гладких векторных полей $\vec{A}(\vec{s})$ на $S^{2}$ введем операции градиента функции $\nabla_{s}: f(\vec{s}) \rightarrow \nabla_{s} f(\vec{s})$ и дивергенции векторного поля $\vec{A}(\vec{s}) \rightarrow$ $\operatorname{div}_{s} \vec{A}(\vec{s})$ (см. [4]). Скалярное произведение векторов $\vec{a}, \vec{b}$ евклидова пространства $T_{\vec{s}}\left(S^{2}\right)$ обозначается через $(\vec{a}, \vec{b})$.

Конкретизируем, каким образом поле $B(\vec{x}, \vec{s}, t)$ зависит от состояния системы:

$$
\vec{B}(\vec{x}, \vec{s}, t)=\vec{B}_{0}(\vec{x}, t)+\vec{B}_{m}(\vec{x}, t)+\vec{B}_{I}(\vec{x}, t)+\vec{B}_{a}(\vec{s}),
$$

где выполняются следующие условия.

1) $\vec{B}_{0}(\vec{x}, t) \in C^{1}\left(X \times[0, T], \mathbb{R}^{3}\right)$ - заданное внешнее магнитное поле.

2) $\vec{B}_{m}(\vec{x}, t)=\nabla_{x} \int_{\Omega}\left(\nabla_{x} 1 /|\vec{x}-\vec{y}|, \vec{M}(\vec{y}, t)\right) d y+4 \pi \vec{M}(\vec{x}, t)$ - магнитноеполе, созданное всеми МДМ с функцией распределения $f(\vec{x}, \vec{s}, t)$ в момент времени $t$, где

$$
\vec{M}(\vec{x}, t)=\int_{S^{2}} \vec{s} f(\vec{x}, \vec{s}, t) d s
$$

- намагниченность данной системы МДМ. Данная зависимость поля дипольных моментов от функции распределения соответствует уравнениям магнитостатики (см. [2]).

В действительности, функция $1 /|\vec{x}-\vec{y}|$, характеризующая парное взаимодействие МДМ, соответствует приближенному закону этого взаимодействия для двух МДМ, достаточно удаленных друг от друга. Взаимодействие двух МДМ, близких друг к другу, имеет более сложньй характер. С другой стороны, сингулярность в интегральном операторе в формуле для магнитного поля является дополнительной трудностью при исследовании соответствующей математической задачи. В соответствии с этим в статье рассматривается парное взаимодействие МДМ с некоторым модельным гладким потенциалом $U(\vec{x}-\vec{y})$, которьй может возникнуть, например, при аппроксимации сингулярного ядра $1 /|\vec{x}-\vec{y}|$ бесконечно гладкими функциями. При этом естественно потребовать, чтобы магнитное поле, определяемое с помощью этого модельного закона взаимодействия, являлось соленоидальным. Чтобы удовлетворить это требование, определим магнитное поле системы МДМ с функцией распределения $f(\vec{x}, \vec{s}, t)$ формулой

$$
\vec{B}_{m}(\vec{x}, t)=\int_{X}\left(4 \pi \vec{s} \omega_{U}(\vec{x}-\vec{y})+\nabla(\vec{s}, \nabla U(\vec{x}-\vec{y}))\right) f(\vec{y}, \vec{s}, t) d y d s,
$$

где функции $\omega_{U}$ и $U$ связаны формулой $\omega_{U}=\Delta U$. Тогда напряженность магнитного поля определяется по формуле

$$
\vec{H}(\vec{x})=\nabla \int_{\Omega}(\nabla U(\vec{x}-\vec{y}), \vec{M}(\vec{y})) d y,
$$

а индукция магнитного поля по формуле

$$
\vec{B}(\vec{x})=\vec{H}(\vec{x})+4 \pi \int_{\Omega} \omega_{U}(\vec{x}-\vec{y}) M(\vec{y}) d y,
$$


что обеспечивает выполнение условия $\operatorname{div} \vec{B}(\vec{x})=0$. Заметим также, что при $\omega(\vec{x})=\delta(\vec{x})$ модельная зависимость магнитного поля от намагниченности совпадает с зависимостью, даваемой уравнениями Максвелла.

3) В настоящей работе предполагается модельная зависимость энергии обменного взаимодействия

$$
E_{I}=\int_{\Omega} \int_{\Omega} \omega_{0}(\vec{x}-\vec{y})(\vec{M}(\vec{x}), \Delta \vec{M}(\vec{y})) d y d x
$$

эффективное поле обменного взаимодействия связано с функцией распределения соотношением

$$
\vec{B}_{I}=J \int_{S^{2}} \Delta \omega_{0}(\vec{x}-\vec{y}) \vec{s} f(\vec{y}, \vec{s}, t) d y d s
$$

где $\omega_{0}(\vec{x}) \in C_{0}^{\infty}\left(B^{3}(0,1),[0,+\infty)\right), \int_{\mathbb{R}^{3}} \omega_{0}(\vec{x}) d x=1, B^{3}(0,1)$ - шар единичного радиуса с центром в нуле в пространстве $\mathbb{R}^{3}, J \in \mathbb{R}$ - константа обменного взаимодействия.

При $\omega_{0}(\vec{x})=\delta(\vec{x})$ формула для обменного поля совпадает с формулой, принятой для описания обменного взаимодействия, например, в уравнении Ландау-Лифшища (см. [2]). Такое определение эффективного поля обменного взаимодействия отражает многие явления магнетизма, однако строгий вывод этого уравнения, по-видимому, отсутствует.

4) $\vec{B}_{a}(\vec{s})=\nabla V(\vec{s})$, где $V(\vec{x}) \in C^{\infty}\left(\mathbb{R}^{3}\right)$. Это эффективное поле выражает факт зависимости энергии $V(\vec{s})$ состояния $\vec{s}$ от ориентации $\vec{s}$ относительно осей кристаллической решетки. Данное определение поля $\vec{B}_{a}(\vec{s})$ соответствует вводимому в монографиях [1], [2] эффективному полю анизатропии кристалла.

Итак, для каждой частицы рассматриваемой системы ее координаты изменяются по закону:

$$
\vec{x}=\mathrm{const}, \quad \frac{\partial \vec{s}(\vec{x}, t)}{\partial t}=[\vec{B}(\vec{x}, \vec{s}, t) \times \vec{s}(\vec{x}, t)]
$$

где $\vec{B}$ определяется по формулам (3). Тогда функция распределения системы из $N$ частищ есть

$$
f^{N}(\vec{x}, \vec{s}, t)=\frac{1}{N} \sum_{i=1}^{N} \delta\left(\vec{x}-\vec{x}_{i}\right) \delta\left(\vec{s}-\vec{s}_{i}\left(\vec{x}_{i}, t\right)\right)
$$

где функция $\vec{s}_{i}\left(\vec{x}_{i}, t\right)$ - решение уравнения (4), и нетрудно проверить, что $f^{N}(\vec{x}, \vec{s}, t)$ является обобщенным решением уравнения (отметим, что вектор $\left[\overrightarrow{B^{N}} \times \vec{s}\right]$ принадлежит касательной плоскости $\left.T_{\vec{s}}\left(S^{2}\right)\right)$ :

$$
\frac{\partial f^{N}}{\partial t}+\left(\nabla_{s} f^{N},\left[\overrightarrow{B^{N}} \times \vec{s}\right]\right)=0
$$

в том смысле, что для любого $\psi \in C^{1}(X)$ справедливо равенство

$$
\frac{d}{d t} \int_{X} \psi(\vec{x}, \vec{s}) f^{N}(\vec{x}, \vec{s}, t) d x d s=\int_{X} f^{N}(\vec{x}, \vec{s}, t)\left(\left[\vec{B}^{N} \times \vec{s}\right], \nabla_{s} \psi(\vec{x}, \vec{s})\right)
$$

Здесь $\vec{B}^{N}(\vec{x}, \vec{s}, t)$ - полное эффективное магнитное поле, соответствующее распределению $N$ частиц в пространстве $X$ с плотностью $f^{N}(\vec{x}, \vec{s}, t)$. 
Следуя подходу, применяемому при вьводе уравнения Власова (см. [5]), заменим систему $N$ частиц, описываемых уравнением (5), некоторой непрерьвной средой, описываемой системой уравнений (6) (см. ниже). Рассмотрим следующую задачу для неизвестной функции распределения $f(\vec{x}, \vec{s}, t)$ :

$$
\begin{gathered}
\frac{\partial f}{\partial t}+\left([\vec{B} \times \vec{s}], \nabla_{s} f\right)=0, \quad(\vec{x}, \vec{s}) \in X, \quad t \in[0, T] \\
f(\vec{x}, \vec{s}, 0)=f_{0}(\vec{x}, \vec{s}) .
\end{gathered}
$$

Индукция магнитного поля $\vec{B}(\vec{x}, \vec{s}, t)$ определяется по формулам (3)

$$
\begin{aligned}
\vec{B}(\vec{x}, \vec{s}, t)= & \vec{B}_{0}(\vec{x}, t)+\nabla V(\vec{s})+\int_{X} w(\vec{x}-\vec{y}) \vec{s} f(\vec{y}, \vec{s}, t) d y d s \\
& +\nabla \int_{X}(\vec{s}, \nabla U(\vec{x}-\vec{y})) f(\vec{y}, \vec{s}, t) d y d s=\vec{B}(\vec{x}, \vec{s}, t ;[f]),
\end{aligned}
$$

где

1) $[0, T]$ - произвольньй отрезок времени, $X$ - описанное выше координатное пространство;

2) $f(\vec{x}, \vec{s}, t) \in C^{1}\left([0, T] \times X, \mathbb{R}^{+}\right)$- неизвестная функция распределения на пространстве $X$ такая, что $f(\vec{x}, \vec{s}, 0)=f_{0}(\vec{x}, \vec{s})$; функция $f_{0}(\vec{x}, \vec{s})$ удовлетворяет условиям: $f_{0}(\vec{x}, \vec{s}) \in C^{1}\left(X, \mathbb{R}^{+}\right), \int_{X} f_{0}(\vec{x}, \vec{s}) d x d s=1 ;$

3) функция $U$ удовлетворяет условиям

$$
\Delta U \in C_{0}^{\infty}\left(B^{3}(0,1),[0,+\infty)\right), \quad \int_{\mathbb{R}^{3}} \Delta U(\vec{x}) d x=1
$$

4) $w(\vec{x})=J \Delta \omega_{0}(\vec{x})+\Delta U(\vec{x}) \in C_{0}^{\infty}\left(\mathbb{R}^{3},[0,+\infty)\right)$ в соответствии с определениями полей магнитного, дипольного и обменного взаимодействий.

Далее функции $U(\vec{x})$ и $w(\vec{x})$ рассматриваются как независимые.

Систему (6) будем назьвать системой уравнений среднего поля.

В данной работе изучается вопрос корректности задачи (6). Кроме того, получен некоторый результат о близости решения задачи (6) и задачи, описьвающей движение конечного числа частиц (5), которьй, в частности, подтверждает применимость системы уравнений (6) к описанию явлений в магнетиках. Для исследования этих проблем автор применяет комбинацию методов изучения корректности задачи Коши для уравнения Власова, предложенных в [5] и [6].

2. Обобщенное решение системы уравнений среднего поля. Дадим следующее

ОПРЕДЕЛЕНИЕ 1. Функция $f(\vec{x}, \vec{s}, t)$, определенная на $X \times[0, T]$, назьвается $\kappa л а с с и-$ ческим решением сглаженной системы (6), если

1) $f(\vec{x}, \vec{s}, t) \in C^{1}\left([0, T] \times X, \mathbb{R}_{+}\right)$;

2) $f(\vec{x}, \vec{s}, t)$ удовлетворяет системе уравнений $(6)$. 
ЗАмЕчАниЕ. Нетрудно проверить, что для классического решения системы (6) интеграл $\int_{X} f(\vec{x}, \vec{s}, t) d x d s$ не зависит от времени. (Поскольку $\operatorname{div}_{s}[\vec{B} \times \vec{s}]=0$.)

Рассмотрим пространство $M$ (не обязательно знакоопределенных) борелевских мер на пространстве $X$. Определим в пространстве $M$ метрику $d$ соотношением: пусть $\mu, \nu \in M ;$ тогда

$$
d(\mu, \nu)=\sup _{f \in D}\left|\int_{X} \mu(d x d s) f(\vec{x}, \vec{s})-\int_{X} \nu(d x d s) f(\vec{x}, \vec{s})\right|
$$

где

$$
D=\{f|f: X \rightarrow[0,1], \quad| f(\vec{x}, \vec{s})-f(\vec{y}, \vec{\sigma})|\leqslant| \vec{x}-\vec{y}|+| \vec{s}-\vec{\sigma} \mid\} .
$$

Тогда метрика $d$ порождает топологию слабой сходимости в пространстве $M$ : для последовательности $\mu_{n} \in M$ условие $\lim _{n \rightarrow \infty} d\left(\mu_{n}, \mu\right)=0$ эквивалентно условию (см. [5], [6])

$$
\lim _{n \rightarrow \infty} \int_{X} \mu_{n}(d x d s) \psi(\vec{x}, \vec{s})=\int_{X} \mu(d x d s) \psi(\vec{x}, \vec{s})
$$

для любого $\psi \in C(X)$.

На фиксированном вьше отрезке $[0, T]$ рассмотрим множество слабо непрерывных отображений $C([0, T], M)$.

ОПРЕДЕЛЕНИЕ 2 . Функция $\mu(t, d x d s) \in C([0, T], M)$ называется обобщенным $р е$ шением системы уравнений среднего поля, если она при любом $\psi(\vec{x}, \vec{s}) \in C^{1}(X)$ удовлетворяет следующей системе уравнений (7) на отрезке $[0, T]$ :

$$
\begin{gathered}
\int_{X} \psi(\vec{x}, \vec{s})(\mu(t, d x d s)-\mu(0, d x d t))=\int_{0}^{t} d \tau \int_{X}\left(\nabla_{s} \psi(\vec{x}, \vec{s}),[\vec{B} \times \vec{s}]\right) \mu(\tau, d x d s),\left(7_{1}\right) \\
\vec{B}(\vec{x}, \vec{s}, t)=\vec{B}_{0}(\vec{x}, t)+\nabla V(\vec{s})+\vec{A}(\vec{x}, t)+\nabla \varphi(\vec{x}, t), \\
\varphi(\vec{x}, t)=\int_{X}(\nabla U(\vec{x}-\vec{y}), \vec{s}) \mu(t, d y d s), \\
\vec{A}(\vec{x}, t)=\int_{X} w(\vec{x}-\vec{y}) \vec{s} \mu(t, d y d s), \\
\mu(+0)=\mu_{0},
\end{gathered}
$$

где $\mu_{0}(d x d s) \in M(X)$ - положительная мера и $\int_{X} \mu_{0}(d x d s)=1$.

Теорема 1. Пусть выполнены следующие предположения о функииях $w(\vec{x}), V(\vec{s})$, $U(\vec{x}), \vec{B}_{0}(\vec{x}, t): \vec{B}_{0}(\vec{x}, t) \in C^{1}\left(X \times[0, T], \mathbb{R}^{3}\right)$ и существует такая положительная постоянная $L$, что

$$
\begin{aligned}
& \left|D_{\vec{x}}^{2} U(\vec{x})\right| \leqslant L, \quad\left|D_{\vec{x}}^{2}(U(\vec{x})-U(\vec{y}))\right| \leqslant L|\vec{x}-\vec{y}| \quad \forall(\vec{x}, \vec{y}) \in \Omega, \\
& |\nabla V(\vec{s})| \leqslant L, \quad|\nabla V(\vec{s})-\nabla V(\vec{\sigma})| \leqslant L|\vec{s}-\vec{\sigma}| \quad \forall(\vec{s}, \vec{\sigma}) \in S^{2}, \\
& |w(\vec{x})| \leqslant L, \quad|w(\vec{x})-w(\vec{y})| \leqslant L|\vec{x}-\vec{y}| \quad \forall(\vec{x}, \vec{y}) \in \mathbb{R}^{3} .
\end{aligned}
$$


Тогда

1) если $\mu_{0}$ - неотричательная борелевская мера такая, что $\int_{X} \mu_{0}(d x d s)=1$, то для любого $T>0$ существует единственное обобщенное решение системы (6), причем для любого $t \in[0, T]$ мера $\mu(t)$ неотричательна и

$$
\int_{X} \mu(t, d x d s)=1
$$

2) существует такое $C>0$, что если $\mu(t)$ и $\nu(t)$ суть обобщенные решения уравнения среднего поля с начальными условиями $\mu_{0}$ и $\nu_{0}$ соответственно, то справедливо неравенство $d(\mu(t), \nu(t)) \leqslant e^{C t} d\left(\mu_{0}, \nu_{0}\right)$ для всех $t \in \mathbb{R}$, $\left\{\mu_{0}, \nu_{0}\right\} \in M(X)$.

ДокАЗАтЕльство. Рассмотрим функцию $\mu(t) \in C([0, T], M)$. Она определяет зависимость от времени поля $\vec{B}_{\mu(\cdot)}(\vec{x}, \vec{s}, t)$ в соответствии с формулами $\left(7_{2}\right)-\left(7_{4}\right)$.

Согласно предположению теоремы относительно функций $U, V, w$ задача Коши

$$
\frac{d \vec{s}(\vec{x}, \vec{\sigma}, t)}{d t}=\left[\vec{B}_{\mu(\cdot)} \times \vec{s}\right], \quad t \in[0, T], \quad \vec{s}(\vec{x}, \vec{\sigma}, 0)=\vec{\sigma}
$$

при любых $(\vec{x}, \vec{\sigma}) \in X$ имеет на $[0, T]$ единственное решение $\vec{s}(\vec{x}, \vec{\sigma}, t \mid \mu(\cdot))$, и ясно, что это решение непрерывно дифференпируемо по $(t, \vec{x}, \vec{\sigma})$, т.е. $\vec{s}(\vec{x}, \vec{\sigma}, t \mid \mu(\cdot)) \in C^{1}([0, T] \times$ $\left.X, S^{2}\right)$. Определим отображение $T_{t}[\mu(\cdot)]$, действующее из $C^{1}(X)$ в $C^{1}(X)$ :

$$
\left(T_{t}[\mu(\cdot)] \psi\right)(\vec{x}, \vec{\sigma})=\psi(\vec{x}, \vec{s}(\vec{x}, \vec{\sigma}, t \mid \mu(\cdot))), \quad t \in[0, T]
$$

Для произвольного $\nu_{0} \in M$ определим функцию $\nu(t) \in C([0, T], M)$ соотношением $\nu(t)=\nu_{0} \circ T_{t}[\mu(\cdot)]$, т.е.

$$
\int_{X} \nu(t, d x d s) \psi(\vec{x}, \vec{s})=\int_{X} \nu_{0}(d x d s)\left(T_{t}[\mu(\cdot)] \psi\right)(\vec{x}, \vec{s})
$$

Тогда справедлива следующая лемма.

ЛЕммА. Функиия $\mu(t) \in C([0, T], M)$ является обобщенным решением уравнения среднего поля с начальным.ми даннымм $\mu_{0}$ тогда и только тогда, когда $\mu(t)$ есть неподвижная точка отобрахсения $\Lambda: \Lambda \mu(t)=\mu_{0} \circ T_{t}[\mu(\cdot)]$.

ДокАЗАТЕЛЬСТво. Если $\mu(t)$ является неподвижной точкой отображения $\Lambda$, то $\mu(t)$, очевидно, удовлетворяет совокупности условий (7).

Пусть функция $\mu(t)$ есть обобщенное решение системы (6). Рассмотрим отображение $S_{t}(\vec{x} \mid \mu(\cdot)) \in C^{1}\left(S^{2}, S^{2}\right): \vec{\sigma} \rightarrow \vec{s}(\vec{x}, \vec{\sigma}, t \mid \mu(\cdot))$, задаваемое задачей (8) при каждом значении $\vec{x}$. Нетрудно проверить, что данное отображение является взаимно однозначньм и якобиан его равен 1. (Чтобы доказать этот факт, достаточно убедится в том, что $\operatorname{div}_{s}[\vec{s} \times \vec{B}(\vec{x}, \vec{s}, t \mid \mu)]=0$.) Следовательно, корректно определено обратное отображение $S_{t}^{-1}(\vec{x} \mid \mu(\cdot)) \in C^{1}\left(S^{2}, S^{2}\right): \vec{\sigma} \rightarrow \vec{n}(\vec{x}, \vec{\sigma}, t \mid \mu(\cdot))$. Поскольку 
$S_{t}^{-1}(\vec{x} \mid \mu(\cdot)) \circ S_{t}(\vec{x} \mid \mu(\cdot))(\vec{\sigma})=\vec{n}(\vec{x}, \vec{s}(\vec{x}, \vec{\sigma}, t \mid \mu(\cdot)), t \mid \mu(\cdot))=\vec{\sigma}$, то функция $\vec{n}(\vec{x}, \vec{\sigma}, t \mid \mu(\cdot))$ при каждом значении $(\vec{x}, \vec{\sigma}) \in X$ и каждом $i=1,2$ является решением задачи Коши

$$
\begin{gathered}
\frac{\partial}{\partial t}(\vec{n}(\vec{x}, \vec{s}, t))_{i}+\left(\nabla_{s}\left(\vec{n}(\vec{x}, \vec{s}, t)_{i}\right),\left[\vec{B}_{\mu(\cdot)}(\vec{x}, \vec{s}, t) \times \vec{s}\right]\right)=0 \\
\vec{n}(\vec{x}, \vec{s}, 0)=\vec{s}
\end{gathered}
$$

которое единственно и принадлежит классу $C^{1}\left(X \times[0, T], S^{2}\right)$ в силу предположений теоремы. Учитывая уравнения $\left(7_{1}\right)$ и $\left(8^{\prime}\right)$, нетрудно показать, что

$$
\frac{d}{d t} \int_{X} \psi(\vec{x}, \vec{n}(\vec{x}, \vec{s}, t \mid \mu(\cdot))) \mu(t, d x d s)=0
$$

для любого $\psi \in C^{1}(X)$. Следовательно, для любого $t \in[0, T]$ и любого $\psi \in C^{1}(X)$ справедливо равенство

$$
\int_{X} \psi(\vec{x}, \vec{\sigma}) \mu_{0}(d x d \sigma)=\int_{X} \psi(\vec{x}, \vec{n}(\vec{x}, \vec{\sigma}, t \mid \mu(\cdot))) \mu(t, d x d \sigma) .
$$

Поскольку отображение $S_{t}^{-1}(\vec{x} \mid \mu(\cdot))$ взаимно однозначное при любых $\vec{x}$ и $t$, то отображение $C^{1}(X) \rightarrow C^{1}(X)$ :

$$
\psi(\vec{x}, \vec{s}) \rightarrow \psi(\vec{x}, \vec{n}(\vec{x}, \vec{s}, t \mid \mu(\cdot)))
$$

является взаимно однозначным преобразованием пространства $C^{1}(X)$. Поэтому для любого обобщенного решения задачи (6) при всех $\psi \in C^{1}(X)$ справедливо соотношение

$$
\int_{X} \psi(\vec{x}, \vec{s}(\vec{x}, \vec{\sigma}, t \mid \mu(\cdot))) \mu_{0}(d x d \sigma)=\int_{X} \psi(\vec{x}, \vec{\sigma}) \mu(t, d x d \sigma) .
$$

Следовательно, для всякого обобщенного решения задачи (6) вьполнено условие $\mu(t)=$ $\mu_{0} \circ T_{t}[\mu(\cdot)]$. Лемма доказана.

$$
\begin{aligned}
& \text { Пусть } \mu(t)=\mu_{0} \circ T_{t}[\mu(\cdot)], \nu(t)=\nu_{0} \circ T_{t}[\nu(\cdot)] . \text { Тогда } \\
& \begin{aligned}
d(\mu(t), \nu(t)) & =d\left(\mu_{0} \circ T_{t}[\mu(\cdot)], \nu_{0} \circ T_{t}[\nu(\cdot)]\right) \\
& \leqslant d\left(\mu_{0} \circ T_{t}[\mu(\cdot)], \mu_{0} \circ T_{t}[\nu(\cdot)]\right)+d\left(\mu_{0} \circ T_{t}[\nu(\cdot)], \nu_{0} \circ T_{t}[\nu(\cdot)]\right) .
\end{aligned}
\end{aligned}
$$

Для второго слагаемого справедлива оценка

$$
\begin{aligned}
d\left(\mu_{0} \circ T_{t}[\nu(\cdot)], \nu_{0} \circ T_{t}[\nu(\cdot)]\right) & =e^{L t} \sup _{f \in D}\left|\int_{X}\left(d \mu_{0}-d \nu_{0}\right) e^{-L t}\left(T_{t}[\nu(\cdot)] f\right)\right| \\
& \leqslant e^{L t} d\left(\mu_{0}, \nu_{0}\right)
\end{aligned}
$$

так как если $f \in D$, то $e^{-L t}\left(T_{t}[\nu(\cdot)] f\right) \in D$. Действительно, в силу уравнения (8)

$$
\left|\frac{d}{d t}(\vec{s}(\vec{x}, \vec{\sigma}, t)-\vec{s}(\vec{y}, \vec{s}, t))\right| \leqslant L(|\vec{s}(\vec{x}, \vec{\sigma}, t)-\vec{s}(\vec{y}, \vec{s}, t)|+|\vec{x}-\vec{y}|) .
$$


Из последнего неравенства и леммы Гронуолла получается требуемое условие.

А для первого слагаемого имеем

$$
\begin{gathered}
d\left(\mu_{0} \circ T_{t}[\mu(\cdot)], \mu_{0} \circ T_{t}[\nu(\cdot)]\right)=\sup _{f \in D}\left|\int_{X} d \mu_{0}\left(T_{t}[\mu(\cdot)] f-T_{t}[\nu(\cdot)] f\right)\right| \\
\leqslant \int_{X} \mu_{0}(d x d \sigma)|\vec{s}(\vec{x}, \vec{\sigma}, t \mid \mu(\cdot))-\vec{s}(\vec{x}, \vec{\sigma}, t \mid \nu(\cdot))|=\lambda(t),
\end{gathered}
$$

поскольку $f \in D$. Следовательно, в силу (9)

$$
\begin{aligned}
\lambda(t) \leqslant & \int_{X} \mu_{0}(d x d \sigma) \mid \int_{0}^{t} d \tau\left[\vec{B}_{\mu(\cdot)}(\vec{x}, \vec{s}(\tau \mid \mu(\cdot)), \tau) \times \vec{s}(\tau \mid \mu(\cdot))\right] \\
& -\int_{0}^{t} d \tau\left[\vec{B}_{\nu(\cdot)}(\vec{x}, \vec{s}(\tau \mid \nu(\cdot)), \tau) \times \vec{s}(\tau \mid \nu(\cdot))\right] \mid \\
\leqslant & \int_{0}^{t} \int_{X} \mu(\tau, d x d s)\left|\left[\vec{B}_{\mu(\cdot)}(\vec{x}, \vec{s}, \tau) \times \vec{s}\right]-\left[\vec{B}_{\nu(\cdot)}(\vec{x}, \vec{s}, \tau) \times \vec{s}\right]\right| \\
& +L \int_{0}^{t} \int_{X} \mu_{0}(d x d s)|\vec{s}(\tau \mid \mu(\cdot))-\vec{s}(\tau \mid \nu(\cdot))| \\
\leqslant & c_{1}(L) \int_{0}^{t} d \tau d(\mu(\tau), \nu(\tau))+L \int_{0}^{t} \lambda(\tau) d \tau .
\end{aligned}
$$

Поэтому с помошью рассуждений, применяемых для доказательства леммы Гронуолла, можно показать, что

$$
\lambda(t) \leqslant c_{1}(L) \int_{0}^{t} d \tau e^{L(t-\tau)} d(\mu(\tau), \nu(\tau)) .
$$

Таким образом,

$$
d(\mu(t), \nu(t)) \leqslant e^{L t} d\left(\mu_{0}, \nu_{0}\right)+c_{1}(L) \int_{0}^{t} d \tau e^{L(t-\tau)} d(\mu(\tau), \nu(\tau))
$$

Из последнего неравенства и леммы Гронуолла вытекает утверждение 2) теоремы 1.

Пусть $\mu_{0}$ - начальное условие задачи (7). Пусть

$$
C_{\mu}=\left\{\mu(t) \mid \mu(t) \in C([0, T], M): \mu(0)=\mu_{0}\right\}
$$

Произвольной функции $\mu(t) \in C_{\mu}$ поставим в соответствие функцию $\Lambda \mu(t)=\mu_{0}$ 。 $T_{t}[\mu(\cdot)]$. Отображение $\Lambda$ отображает пространство $C_{\mu}$ в себя. Введем в пространстве $C_{\mu}$ метрику: для любых $\mu, \nu \in C_{\mu}$ положим

$$
d_{\alpha}(\mu(\cdot), \nu(\cdot))=\sup _{t \in[0, T]} d(\mu(t), \nu(t)) e^{-\alpha t}, \quad \alpha \in \mathbb{R}
$$

Так как $(M, d)$ есть полное метрическое пространство (см. [6]), то метрическое пространство $\left(C_{\mu}, d_{\alpha}\right)$ также является полным. 
Докажем, что отображение $\Lambda$ пространства $\left(C_{\mu}, d_{\alpha}\right)$ в себя при достаточно больших $\alpha$ является сжатием. Действительно, согласно сделанной выше оценке имеем

$$
\begin{aligned}
d((\Lambda \mu(\cdot))(t),(\Lambda \nu(\cdot))(t)) & =d\left(\mu_{0} \circ T_{t}[\mu(\cdot)], \mu_{0} \circ T_{t}[\nu(\cdot)]\right) \\
& \leqslant c_{1}(L) \int_{0}^{t} d \tau e^{L(t-\tau)} d(\mu(\tau), \nu(\tau)) .
\end{aligned}
$$

Следовательно, при $\alpha>L$

$$
d_{\alpha}(\Lambda \mu(\cdot), \Lambda \nu(\cdot)) \leqslant \frac{c_{1}}{\alpha-L} d_{\alpha}(\mu(\cdot), \nu(\cdot)),
$$

что и доказьвает утверждение.

Поэтому отображение $\Lambda$ имеет единственную неподвижную точку $\mu(t) \in C_{\mu}$. Следовательно, существует единственное обобщенное решение уравнения среднего поля.

Положим в $(9) \psi \equiv 1$. Тогда получим, что для любого $t \in[0, T]$ мера $\mu(t)$ является вероятностной. Теорема 1 доказана.

3. Классическое решение уравнения среднего поля. В этом пункте приводится исследование гладкости решения задачи (7) при условии, что начальная мера обладает гладкой плотностью.

Рассмотрим задачу (7) с начальной мерой $\mu_{0}(d x d s)=f_{0}(\vec{x}, \vec{s}) d x d s$, где функция $f_{0}(\vec{x}, \vec{s}) \in C^{1}(X)$ - плотность неотрищательной нормированной меры.

Теорема 2. Пусть выполнены предположения теоремы 1 о свойствах функиий $U, V$ и w. Пусть функиия $f_{0}(\vec{x}, \vec{s})$ принадлежит пространству $C^{1}(X)$. Тогда на отрезке $[0, T]$ существует единственное классическое решение задачи Коuи (6).

ДокАЗАтЕЛЬСтво. Докажем сушествование классического решения. Пусть $\mu(t)-$ обобщенное решение задачи (6) с начальной мерой $\mu_{0}(d x d s)=f_{0}(\vec{x}, \vec{s}) d s d x$. По теореме 1 это решение существует и единственно. В силу предположений теоремы поля $\vec{B}_{\mu(\cdot)}(\vec{x}, \vec{s}, t)$, отвечающие этому решению, принадлежат пространству $C^{1}(X \times[0, T]$, $\left.\mathbb{R}^{3}\right)$. Следовательно, задача Коши (8) имеет единственное решение $\vec{s}(\vec{x}, \vec{\sigma}, t \mid \mu(\cdot)) \in$ $C^{1}\left(X \times[0, T], S^{2}\right)$.

В соответствии с утверждением леммы $\mu(t)=\mu \circ T_{t}[\mu(\cdot)]$, поэтому для любого $\psi \in$ $C^{1}(X)$ справедливо равенство

$$
\int_{X} \mu(t, d x d s) \psi(\vec{x}, \vec{s})=\int_{X} f_{0}(\vec{x}, \vec{s}) d x d \sigma \psi(\vec{x}, \vec{s}(\vec{x}, \vec{\sigma}, t \mid \mu(\cdot))) .
$$

Отображение $S_{t}^{-1}(\vec{x} \mid \mu(\cdot)): \vec{\sigma} \rightarrow \vec{n}(\vec{x}, \vec{\sigma}, t \mid \mu(\cdot))$ при любых $\vec{x} \in \Omega$ и $t \in[0, T]$ является взаимно однозначным отображением с равным единице якобианом (см. лемму), поэтому если в последнем равенстве сделать замену переменных интегрирования $\vec{\sigma} \rightarrow \vec{n}(\vec{x}, \vec{\sigma}, t \mid \mu(\cdot))$, то равенство примет вид

$$
\int_{X} \mu(t, d x d s) \psi(\vec{x}, \vec{s})=\int_{X} f_{0}(\vec{x}, \vec{n}(\vec{x}, \vec{\sigma}, t \mid \mu(\cdot))) \psi(\vec{x}, \vec{\sigma}) d x d \sigma .
$$


Поскольку функция $\vec{n}(\vec{x}, \vec{\sigma}, t \mid \mu(\cdot))$ является решением задачи Коши $\left(8^{\prime}\right)$, то в силу предположений теоремы о функциях $U, V$ и $w$ она принадлежит пространству $C^{1}(X \times$ $\left.[0, T], S^{2}\right)$.

Следовательно, обобщенное решение задачи (6) $\mu(t)$ имеет плотность $f(\vec{x}, \vec{\sigma}, t)=$ $f_{0}(\vec{x}, \vec{n}(\vec{x}, \vec{\sigma}, t \mid \mu(\cdot)))$ и $f(\vec{x}, \vec{s}, t) \in C^{1}\left(X \times[0, T], \mathbb{R}^{+}\right)$. Тогда

$$
\int_{X} \psi(\vec{x}, \vec{s})\left(\frac{\partial f}{\partial t}-\left(\nabla_{s} f,[\vec{B} \times \vec{s}]\right)\right) d x d s=0
$$

для произвольного $\psi \in C^{1}(X)$, поэтому функция $f(\vec{x}, \vec{s}, t)$ удовлетворяет системе уравнений (6) и, следовательно, является классическим решением задачи (6).

Единственность этого решения следует из единственности решения задачи Коши $\left(8^{\prime}\right)$. Теорема 2 доказана.

4. Обсуждение результатов. Предположим, что мера $\mu_{N}(0)$ определена формулой

$$
\int_{X} \psi d \mu_{N}(0)=\frac{1}{N} \sum_{1 \leqslant j \leqslant N} \psi\left(\vec{x}_{j}, \vec{s}_{j}\right),
$$

где $\vec{x}_{j}, \vec{s}_{j}, j=1, \ldots, N,-$ набор точек в пространстве $X$.

Тогда решение системы $(7)$ с начальной мерой $\mu_{N}(0)$ существует и как неподвижная точка отображения $\Lambda$ определяется формулой

$$
\int \psi d \mu_{N}(t)=\frac{1}{N} \sum_{1 \leqslant j \leqslant N} \psi\left(\vec{x}_{j}, \vec{s}^{N}\left(\vec{x}_{j}, \vec{s}_{j}, t\right)\right)
$$

где $\vec{s}^{N}\left(\vec{x}_{j}, \vec{s}_{j}, t\right)$ есть решение системы уравнений

$$
\begin{aligned}
& \vec{s}^{N}\left(\vec{x}_{j}, \vec{s}_{j}, 0\right)=\vec{s}_{j}, \\
& D_{t} \vec{s}^{N}\left(\vec{x}_{j}, \vec{s}_{j}, t\right)=\left(\left(\nabla_{x_{j}} \frac{1}{N} \sum_{1 \leqslant i \leqslant N}\left(\nabla_{x_{j}} U\left(\vec{x}_{j}-\vec{x}_{i}\right), \vec{s}^{N}\left(\vec{x}_{i}, \vec{s}_{i}, t\right)\right)\right.\right. \\
&+\frac{1}{N} \sum_{1 \leqslant i \leqslant N} w\left(\vec{x}_{j}-\vec{x}_{i}\right) \vec{s}^{N}\left(\vec{x}_{i}, \vec{s}_{i}, t\right)+\nabla V\left(\vec{s}^{N}\left(\vec{x}_{j}, \vec{s}_{j}, t\right)\right) \\
&\left.\left.+\vec{B}_{0}\left(\vec{x}_{j}, t\right)\right) \times \vec{s}^{N}\left(\vec{x}_{j}, \vec{s}_{j}, t\right)\right) .
\end{aligned}
$$

Данная система уравнений является системой уравнений движения $N$ частиц, положение в пространстве которых $\vec{x}_{i}, i=1, \ldots, N$, фиксированно, а механический момент $\vec{s}_{i}^{N}(t)$ изменяется по закону $D_{t} \vec{s}_{i}^{N}(t)=\vec{F}_{i}^{N}(t) ; \vec{F}_{i}^{N}(t)-$ момент сил, действующих на частицу.

Изменение механического момента $\vec{s}_{i}^{N}$ частицы обусловленно тем, что с ним связан дипольньй магнитньй момент $\vec{m}_{i}^{N}=\mu \vec{s}_{i}^{N}=\vec{s}_{i}^{N}$. Система $N$ частицпредставляет собой набор находящихся в поле друг друга магнитных диполей, и момент сил магнитного дипольного взаимодействия $i$-й частицы с другими частицами равен

$$
\vec{F}_{\mathrm{mag}}^{N}(t)=\left[\vec{B}_{\mathrm{mag}}^{N}\left(\vec{x}_{i}, t\right) \times \vec{s}_{i}^{N}\right]
$$


В формуле (10) кроме сил магнитного дипольного взаимодействия частиц с помощью эффективного магнитного поля учтено также взаимодействие каждого электрона с атомами кристаллической решетки как целым (слагаемое $V(s))$ и обменноевзаимодействие соседних электронов.

Пусть $\mu_{\infty}(t)$ - обобщенное решение задачи (6) с начальным условием $\mu_{\infty}(0)=\mu_{\infty}$. Тогда из теоремы 1 следует, что если $\lim _{N \rightarrow \infty} d\left(\mu_{N}, \mu_{\infty}\right)=0$, то на произвольном отрезке $[0, T]$ решение $\mu_{N}(t)$ задачи $(7)$ с начальным условием $\mu_{N}$ сходится к решению системы (7) с начальной мерой $\mu_{\infty}$ в пространстве $C([0, T], M)$ с метрикой $d_{\alpha}$. Если мера $\mu_{\infty}(t)$ имеет гладкую плотность

$$
f(\vec{x}, \vec{s}, t)=w *-\lim _{N \rightarrow \infty} \frac{1}{N} \sum_{1 \leqslant j \leqslant N} \delta\left(\vec{x}-\vec{x}_{j}\right) \delta\left(\vec{s}-\vec{s}\left(\vec{x}_{j}, \vec{s}_{j}, t\right)\right)
$$

то эта плотность является классическим решением приближенного уравнения (6) и содержит информацию о движении реальной спиновой системы с функцией распределения $f_{N}(\vec{x}, \vec{s}, t)$. В этом смысле уравнение $(6)$, описьвающее движение непрерьвной системы, является “власовским" пределом уравнения движения системы $N$ частиц (10) при $N \rightarrow \infty$ (см. [5]).

Пользуюсь возможностью выразить искреннюю благодарность моему научному руководителю П. Е. Жидкову за постановку задачи, полезные обсуждения и внимание к работе.

\section{СПИСОК ЦИТИРОВАННОЙ ЛИТЕРАТУРЫ}

[1] Ахиезер А. И., Берестецкий В. Г., Пелетминский С. В. Спиновые волны. М.: Наука, 1967.

[2] О'Делл Т. Ферромагнитодинамика. М.: Мир, 1983.

[3] Парселл Э. Электричество и магнетизм. М.: Наука, 1983.

[4] Постников М. М. Гладкие многообразия. М.: Наука, 1987.

[5] Арсеньев А. А. Лекции о кинетических уравнениях. М.: Наука, 1992.

[6] Spohn H. Large-Scale Dynamics on Interacting Particle. New York: Springer-Verlag, 1991.

Московский физико-технический институт 